\title{
Moment Problem and Inverse Cauchy Problems for Heat Equation
}

\author{
O. Yaremko, N.Yaremko*, T. Eliseeva \\ Penza State University, Penza, Russia \\ *Corresponding author: yaremki@mail.ru
}

Received January 20, 2015; Revised February 22, 2015; Accepted March 02, 2015

\begin{abstract}
The solution of Hamburger and Stieltjes moment problem can be thought of as the solution of a certain inverse Cauchy problem. The solution of the inverse Cauchy problem for heat equation is founded in the form of Hermite polynomial series. The author reveals, the formulas obtained by him for the solution of inverse Cauchy problem have a symmetry with respect to the formulas for corresponding direct Cauchy problem. Obtained formulas for solution of the inverse problems can serve as a basis for the solution of the moment problem.
\end{abstract}

Keywords: hamburger and stieltjes moment problem, inverse cauchy problem, hermite polynomials, poisson integral

Cite This Article: O. Yaremko, N.Yaremko, and T. Eliseeva, "Moment Problem and Inverse Cauchy Problems for Heat Equation." Turkish Journal of Analysis and Number Theory, vol. 3, no. 1 (2015): 33-36. doi: 10.12691/tjant-3-1-8.

\section{Introduction}

Motivated by considerations arising from many-body quantum physics the authors consider the moment problem in the general case where the moments are finite real numbers. They present a well- defined analytic procedure for the construction of an infinite set of exact solutions to the above problem and discuss several special cases. Before the development of Quantum Mechanics, mathematicians were interested in understanding the relationship between a positive function, $f(x)$ and its (power) moments,

$$
m_{j}=\int_{I} \xi^{j} d f(\xi), j=0,1,2, \ldots
$$

$j \geq 0$ and integer. The basic question was, what properties must all of the moments have in order to conclude that the underlying function is positive, or nonnegative (i.e., $f(x)>0$ This is referred to as the Moment Problem $[1,7,8]$. Depending on the nature of the domain, $I$, on which the function is defined, the corresponding Moment Problem is given different names. Thus, if $I$ the entire real axis, we call this the Hamburger moment problem, in recognition of the mathematician Hamburger who proved a particular theorem, quoted below. If $I=R_{+}$, the nonnegative real axis (including zero), then we refer to the Stieltjes Moment Problem, in recognition of Stieltjes. This particular form of the Moment Problem is very important because it is related to the theory of Pade approximants [6]. If $I=[0,1]$, the closed unit interval, then we say that we have a Hausdorff Moment Problem. Finally, if one deals with periodic functions,

$$
f(x+2 \pi)=f(x)
$$

then one has the Trigonometric moment problem, in which the moments are defined in terms of periodic functions

$$
m_{j}=\int_{0}^{2 \pi} e^{i j \xi} d f(\xi), j=0, \pm 1, \pm 2, \ldots
$$

Another issue is, suppose one is given the infinite set of numbers, $m_{j}$, when are these the moments of a unique positive function? That is, when can we guarantee that there are no other positive functions having the same moments? This is an important concern, and leads to the Carlemann conditions for uniqueness [7]. Although the Stieltjes moment problem was solved in the 1800s. In early 1900's, Markov [7] obtained significant results for both the feasibility and the bounding moment problems. Later, in 1920, Hamburger [8] extended the Stieltjes moment problem to the real axis, and established the moment problem as a theory of its own. In the same time, Hausdorff [8], defined the Hausdorff moment problem on a finite interval in connection with convergencepreserving matrices; this new approach for the moment problem was the first one not related to continued fractions. Two years later, in 1922, Nevanlinna [8] extended the Hamburger moment problem to the complex functions. Riesz in 1923 [8], was the first who extended the moment problem in functional analysis, by observing the connection between the moment problem and the space of bounded linear functional on $\mathrm{C}([\mathrm{a}, \mathrm{b}])$.

The Hamburger moment problem is solvable (that is, $\left\{m_{n}\right\}$ is a sequence of moments) if and only if the corresponding Hankel kernel on the nonnegative integers 
is positive definite, i.e.,

$$
A=\left(\begin{array}{cccc}
m_{0} & m_{1} & m_{2} & \ldots \\
m_{1} & m_{2} & m_{3} & \ldots \\
m_{2} & m_{3} & m_{3} & \ldots \\
\cdots & \ldots & \ldots & \ldots
\end{array}\right)
$$

$$
\sum_{j, k \geq 0} m_{j+k} c_{j} \bar{c}_{k} \geq 0
$$

for an arbitrary sequence $c_{j}, \quad j \geq 0$ of complex numbers with finite support (i.e. $c_{j}$ except for finitely many values of $j$ ).

In this paper a moment problem reduces to the inverse Cauchy problems for the heat equation. The inverse Cauchy problem for the heat equation consists of to reconstruct a priori unknown initial condition of the dynamic system from its known final condition.

The inverse Cauchy problem solution is found in the Hermite polynomial series form. We noticed the solution of inverse Cauchy problem possess the symmetry with respect to solution of the direct Cauchy problem.

The principal result of the article is the solution of the moment problem regarded as the solution of a certain inverse Cauchy problem.

\section{The Inverse Cauchy Problem for the Heat Equation on the Real Axis}

The inverse problem for the heat equation of an infinite bar is to find the unknown initial distribution $f(x)$ of thermal field by the known thermal field $u(\tau, x)$ $([1,2,5,8,9])$. This problem leads to the solving of first type Fredholm integral equation:

$$
\begin{aligned}
& \int_{-\infty}^{\infty} \frac{1}{2 \sqrt{\pi \tau}} \exp \left(-\frac{(x-\xi)^{2}}{4 \tau}\right) f(\xi) d \xi=u(\tau, x), \\
& \tau>0, x \in R .
\end{aligned}
$$

The left side of equation (10) is the Poisson's integral, [10]. As it is shown in [10] the solution of equation (10) is:

$$
f(x)=\frac{1}{\sqrt{\pi}} \sum_{j=0}^{\infty} \frac{u^{(j)}(0)}{(2 \sqrt{\tau})^{n+1} j !} H_{j}\left(\frac{x}{2 \sqrt{\tau}}\right),
$$

where $H_{j}(z)$ - Hermite polynomials :

$$
H_{j}(z)=(-1)^{j} e^{z^{2}} \frac{d^{j}}{d z^{j}}\left(e^{-z^{2}}\right) .
$$

We get a solution of equation (4) by the Fourier transform integral method from $[10,11]$

$$
f(x)=\frac{1}{2 \pi} \int_{-\infty}^{\infty} e^{\lambda^{2} \tau} e^{i \lambda x}\left(\int_{-\infty}^{\infty} e^{-i \lambda \xi} d \xi\right) d \lambda .
$$

If $\beta>0$, then the last formula takes the form

$$
f(x)=\frac{1}{2 \pi} \int_{-\infty}^{\infty} e^{-\lambda^{2} \beta} e^{\lambda^{2}(\tau+\beta)} e^{i \lambda x}\left(\int_{-\infty}^{\infty} e^{-i \lambda \xi} u(\tau, \zeta) d \xi\right) d \lambda .
$$

The function $e^{\lambda^{2}(\tau+\beta)+i \lambda x}$ is a generating function for the Hermite polynomials, this means that

$$
e^{\lambda^{2}(\tau+\beta)-i \lambda x}=\sum_{j=0}^{\infty} \frac{(-i \lambda)^{j}(\tau+\beta)^{\frac{j}{2}}}{j !} H_{j}\left(\frac{\xi}{2 \sqrt{\tau+\beta}}\right)
$$

Because of formula (8) we get

$$
\begin{gathered}
f(x)=\frac{1}{2 \pi} \int_{-\infty}^{\infty} e^{-\lambda^{2} \beta} e^{i \lambda x} \sum_{j=0}^{\infty} \frac{(-i \lambda)^{j}}{j !}(\tau+\beta)^{\frac{j}{2}} \\
\cdot \int_{-\infty}^{\infty} H_{j}\left(\frac{\xi}{2 \sqrt{\tau+\beta}}\right) u(\tau, \xi) d \xi d \lambda .
\end{gathered}
$$

We will change the order of integration and calculate the inner integral with respect to $\lambda$. The Poisson integral

$$
\frac{1}{2 \pi} \int_{-\infty}^{\infty} e^{-\lambda^{2} \beta} e^{i \lambda x} d \lambda=\frac{e^{-\frac{x^{2}}{4 \beta}}}{2 \sqrt{\pi \beta}} .
$$

is used in this calculations.

We calculate the value of an integral by $j$ - time differentiating with respect to $x$ :

$$
\frac{1}{2 \pi} \int_{-\infty}^{\infty}(-i \lambda)^{j} e^{-\lambda^{2} \beta} e^{i \lambda x} d \lambda=(-1)^{j} \frac{d^{j}}{d x^{j}}\left[\frac{e^{-\frac{x^{2}}{4 \beta}}}{2 \sqrt{\pi \beta}}\right] .
$$

On the basis of (6) we can write

$$
\begin{aligned}
& \frac{1}{2 \pi} \int_{-\infty}^{\infty}(-i \lambda)^{j} e^{-\lambda^{2} \beta} e^{i \lambda x} d \lambda \\
& =\frac{e^{-\frac{x^{2}}{4 \beta}}}{2 \sqrt{\pi \beta}} \frac{1}{(2 \sqrt{\beta})^{j}} H_{j}\left(\frac{x}{2 \sqrt{i \beta}}\right) .
\end{aligned}
$$

Finally, first new formula for the initial distribution of the thermal field takes the form

$$
f(x)=\frac{e^{-\frac{x^{2}}{4 \beta}}}{2 \sqrt{\pi \beta}} \sum_{j=0}^{\infty} \frac{1}{(2 \sqrt{\beta})^{j}} H_{j}\left(\frac{x}{2 \sqrt{\beta}}\right) \frac{(\tau+\beta)}{j !} u_{j},
$$

where

$$
u_{j}=\int_{-\infty}^{\infty} H_{j}\left(\frac{\xi}{2 \sqrt{\tau+\beta}}\right) u(\tau, \xi) d \xi
$$

\section{Hamburger Moment Problem}

Let number sequence $\left\{f_{j}\right\}$ defined by the formula

$$
f_{j}=\int_{-\infty}^{\infty} \xi^{j} f(\xi) d \xi, j=0,1,2, \ldots
$$

then the equality 


$$
\begin{aligned}
& \int_{-\infty}^{\infty} \frac{1}{2 \sqrt{\pi \tau}} \exp \left(-\frac{(x-\xi)^{2}}{4 \tau}\right) f(\xi) d \xi \\
& =\int_{-\infty}^{\infty} \frac{\exp \left(-\frac{x^{2}}{4 \tau}\right)}{2 \sqrt{\pi \tau}} \sum_{j=0}^{\infty} \frac{H_{j}\left(\frac{x}{2 \sqrt{\tau}}\right)}{j !} f(\xi) d \xi \\
& =\frac{1}{2 \sqrt{\pi \tau}} \exp \left(-\frac{x^{2}}{4 \tau}\right) \sum_{j=0}^{\infty} \frac{H_{j}\left(\frac{x}{2 \sqrt{\tau}}\right)}{j !} \frac{m_{j}}{(2 \sqrt{\tau})^{j}}
\end{aligned}
$$

is fulfilled. Let us denote function $F(x)$ by equality

$$
\int_{-\infty}^{\infty} \frac{\exp \left(-\frac{x^{2}}{4 \tau}\right)}{2 \sqrt{\pi \tau}} \sum_{j=0}^{\infty} \frac{H_{j}\left(\frac{x}{2 \sqrt{\tau}}\right)}{j !} \frac{\xi^{j}}{(2 \sqrt{\tau})^{j}} f(\xi) d \xi=F(x) .
$$

The inverse problem for the heat equation of an infinite bar is consider

$$
\int_{-\infty}^{\infty} \frac{1}{2 \sqrt{\pi \tau}} \exp \left(-\frac{(x-\xi)^{2}}{4 \tau}\right) f(\xi) d \xi=F(x) .
$$

Because of formula (9) we get solution of the Hamburger truncated moment problem of the form

$$
f(x)=\frac{e^{-\frac{x^{2}}{4 \beta}}}{2 \sqrt{\pi \beta}} \sum_{j=0}^{\infty} \frac{1}{(2 \sqrt{\beta})^{j}} H_{j}\left(\frac{x}{2 \sqrt{\beta}}\right) \frac{(\tau+\beta)^{\frac{j}{2}}}{j !} F_{j},(10)
$$

where

$$
F_{j}=\int_{-\infty}^{\infty} H_{j}\left(\frac{\xi}{2 \sqrt{\tau+\beta}}\right) F(\xi) d \xi
$$

\section{The Inverse Cauchy Problem for the Heat Equation on the Nonnegative Real Axis}

The inverse problem for the heat equation of an half infinite bar leads to the solving of first - type Fredholm integral equation:

$$
\int_{0}^{\infty} G(\tau, x, \xi) f(\xi) d \xi=u(\tau, x)
$$

where

$$
G(\tau, x, \xi)=\frac{1}{2 \sqrt{\pi \tau}}\left(\exp \left(-\frac{(x-\xi)^{2}}{4 \tau}\right)-\exp \left(-\frac{(x+\xi)^{2}}{4 \tau}\right)\right) .
$$

The left side of equation (11) is the Poisson's integral, [10]. We get a solution of equation (11) by the sintransform integral method $[11,12]$

$$
f(x)=\frac{1}{2 \pi} \int_{-\infty}^{\infty} e^{\lambda^{2} \tau} e^{i \lambda x}\left(\int_{-\infty}^{\infty} e^{-i \lambda \xi} u(\tau, \xi) d \xi\right) d \lambda .
$$

If $\beta>0$, then the last formula takes the form $f(x)=\frac{2}{\pi} \int_{0}^{\infty} e^{-\lambda^{2} \beta} e^{\lambda^{2}(\tau+\beta)} \sin \lambda x\left(\int_{0}^{x} \sin \lambda \xi u(\tau, \xi) d \xi\right) d \lambda$.

The function $e^{\lambda^{2}(\tau+\beta)+i \lambda x}$ is a generating function for the Hermite polynomials, this means that

$$
\begin{aligned}
& e^{\lambda^{2}(\tau+\beta)} \sin \lambda \xi=-\operatorname{Im}\left[e^{\left.\lambda^{2}(\tau+\beta)-i \lambda \xi\right]}\right. \\
& =-\operatorname{Im} \sum_{j=0}^{\infty} \frac{(-i \lambda)^{j}(\tau+\beta)^{\frac{j}{2}}}{j !} H_{j}\left(\frac{\xi}{2 \sqrt{\tau+\beta}}\right) \\
& =\sum_{j=0}^{\infty} \sin \frac{\pi}{2} j \frac{\lambda^{j}(\tau+\beta)^{\frac{j}{2}}}{j !} H_{j}\left(\frac{\xi}{2 \sqrt{\tau+\beta}}\right) \\
& =\sum_{j=1,3,5, \ldots}^{\infty} \sin \frac{\pi}{2} j \frac{\lambda^{j}(\tau+\beta)^{\frac{j}{2}}}{j !} H_{j}\left(\frac{\xi}{2 \sqrt{\tau+\beta}}\right) .
\end{aligned}
$$

Because of formula (12) we get

$$
\begin{gathered}
f(x)=\frac{2}{\pi} \int_{0}^{\infty} e^{-\lambda^{2} \beta} \sin \lambda x \\
\cdot \sum_{j=1,3,5, \ldots}^{\infty} \sin \frac{\pi}{2} j \frac{\lambda^{j}(\tau+\beta)^{\frac{j}{2}}}{j !} \\
\cdot \int_{0}^{\infty} H_{j}\left(\frac{\xi}{2 \sqrt{\tau+\beta}}\right) u(\tau, \xi) d \xi d \lambda .
\end{gathered}
$$

We will change the order of integration and calculate the inner integral with respect to $\lambda$. The Poisson integral is used in this calculations. We get

$$
\frac{1}{2 \pi} \int_{-\infty}^{\infty} e^{-\lambda^{2} \beta} e^{i \lambda x} d \lambda=\frac{e^{-\frac{x^{2}}{4 \beta}}}{2 \sqrt{\pi \beta}}
$$

We calculate the value of an integral by $j$ - time differentiating with respect to $x$ :

$$
\begin{gathered}
\frac{2}{\pi} \int_{0}^{\infty} \sin \frac{\pi}{2} j \lambda^{j} e^{-\lambda^{2} \beta} \sin \lambda x d \lambda=(-1)^{j} \frac{d^{j}}{d x^{j}}\left[\frac{e^{-\frac{x^{2}}{4 \beta}}}{2 \sqrt{\pi \beta}}\right], \\
\sin \frac{\pi}{2} j=(-1)^{\frac{j-1}{2}}, j=1,3,5, \ldots \\
\frac{2}{\pi} \int_{0}^{\infty} \lambda^{j} e^{-\lambda^{2} \beta} \sin \lambda x d \lambda=(-1)^{\frac{j+1}{2}} \frac{d^{j}}{d x^{j}}\left[\frac{e^{-\frac{x^{2}}{4 \beta}}}{2 \sqrt{\pi \beta}}\right], \\
j=1,3,5, \ldots
\end{gathered}
$$

On the basis of (6) we can write

$$
\frac{2}{\pi} \int_{0}^{\infty} \lambda^{j} e^{-\lambda^{2} \beta} \sin \lambda x d \lambda=\frac{e^{-\frac{x^{2}}{4 \beta}}}{2 \sqrt{\pi \beta}} \frac{(-1)^{\frac{j+1}{2}}}{(2 \sqrt{\beta})^{j}} H_{j}\left(\frac{x}{2 \sqrt{\beta}}\right) .
$$


Finally, first new formula for the initial distribution of the thermal field takes the form

$f(x)=\frac{e^{-\frac{x^{2}}{4 \beta}}}{2 \sqrt{\pi \beta}} \sum_{j=1,3,5, \ldots}^{\infty} \frac{(-1)^{\frac{j+1}{2}}}{(2 \sqrt{\beta})^{j}} H_{j}\left(\frac{x}{2 \sqrt{\beta}}\right) \frac{(\tau+\beta)^{\frac{j}{2}}}{j !} u_{j}$,

where

$$
u_{j}=\int_{0}^{\infty} H_{j}\left(\frac{\xi}{2 \sqrt{\tau+\beta}}\right) f(\xi) d \xi
$$

\section{Stieltjes Moment Problem}

Let number sequence $\left\{f_{j}\right\}$ defined by the formula

$$
m_{j}=\int_{0}^{\infty} \xi^{j} f(\xi) d \xi, j=0,1,2, \ldots
$$

then

$$
\begin{aligned}
& \int_{0}^{\infty} \frac{1}{2 \sqrt{\pi \tau}}\left(\exp \left(-\frac{(x-\xi)^{2}}{4 \tau}\right)\right)-\exp \left(-\frac{(x+\xi)^{2}}{4 \tau}\right) f(\xi) d \xi \\
& =\int_{-\infty}^{\infty} \frac{1}{2 \sqrt{\pi \tau}} \exp \left(-\frac{x^{2}}{4 \tau}\right) \\
& \text { • } \sum_{j=1,3,5, \ldots}^{\infty}(-1)^{\frac{j+1}{2}} \frac{H_{j}\left(\frac{x}{2 \sqrt{\tau}}\right)}{j !} f(\xi) d \xi \\
& =\frac{1}{2 \sqrt{\pi \tau}} \exp \left(-\frac{x^{2}}{4 \tau}\right) \sum_{j=1,3,5, \ldots}^{\infty}(-1)^{\frac{j+1}{2}} \frac{H_{j}\left(\frac{x}{2 \sqrt{\tau}}\right)}{j !} \frac{m_{j}}{(2 \sqrt{\tau})^{j}}
\end{aligned}
$$

let us denote this function by

$$
\frac{1}{2 \sqrt{\pi \tau}} \exp \left(-\frac{x^{2}}{4 \tau}\right) \sum_{j=1,3,5, \ldots}^{\infty} \frac{H_{j}\left(\frac{x}{2 \sqrt{\tau}}\right)}{j !} \frac{(-1)^{\frac{j+1}{2}} m_{j}}{(2 \sqrt{\tau})^{j}}=F(x) .
$$

We will consider the inverse problem for the heat equation of an infinite bar

$$
\int_{0}^{\infty} G(\tau, x, \xi) f(\xi) d \xi=F(x),
$$

where

$$
G(\tau, x, \xi)=\frac{1}{2 \sqrt{\pi \tau}}\left(\exp \left(-\frac{(x-\xi)^{2}}{4 \tau}\right)\right)-\exp \left(-\frac{(x+\xi)^{2}}{4 \tau}\right)
$$

Because of formula (14) we get solution of the Hamburger truncated moment problem of the form

$$
f(x)=\frac{e^{-\frac{x^{2}}{4 \beta}}}{2 \sqrt{\pi \beta}} \sum_{j=1,3,5, \ldots}^{\infty} \frac{(-1)^{\frac{j+1}{2}}}{(2 \sqrt{\beta})^{j}} H_{j}\left(\frac{x}{2 \sqrt{\beta}}\right) \frac{(\tau+\beta)^{\frac{j}{2}}}{j !} F_{j},
$$

where

$$
F_{j}=\int_{0}^{\infty} H_{j}\left(\frac{\xi}{2 \sqrt{\tau+\beta}}\right) f(\xi) d \xi, j=1,3,5, \ldots
$$

\section{References}

[1] Akhiezer, N.I., Krein, M.G. Some Questions in the Theory of Moments, Amer. Math. Soc., 1962.

[2] Alifanov, O.M., Inverse problems of heat exchange, M, 1988, p. 279.

[3] Arfken, G. B.; Weber, H. J. (2000), Mathematical Methods for Physicists (5th ed.), Boston, MA: Academic Press.

[4] Bavrin, I. I., Yaremko, O. E. Transformation Operators and Boundary Value Problems in the Theory of Harmonic and Biharmonic Functions (2003) Doklady Mathematics, 68 (3), pp. 371-375.

[5] Baker, G. A., Jr.; and Graves-Morris, P. Padé Approximants. Cambridge U.P., 1996.

[6] Beck, J.V., Blackwell, V., Clair, C.R., Inverse Heat Conduction. Ill-Posed Problems, M, 1989, p. 312.

[7] Chebysev, P. Sur les valeurs limites des intégrales, Journal de Mathématiques pures et appliquées, 19 (1874), 157-160.

[8] Krein, M.G. and Nudelman, A.A. The Markov Moment Problem and Extermal Problems, Translations of Mathematical Monographs, Volume Fifty, Library of Congress Cataloging in Publication Data, 1977.

[9] Lavrentev, M.M., Some ill-posed problems of mathematical physics, Novosibirsk, AN SSSR, 1962, p. 92.

[10] Mors, F.M., Fishbah, G. Methods of theoretical physics, 1958.

[11] Yaremko, O.E. Matrix integral Fourier transforms for problems with discontinuous coefficients and transformation operators (2007) Doklady Mathematics, 76 (12), pp. 323-325.

[12] Yaremko, O.E. Transformation operator and boundary value problems Differential Equation. Vol.40, No. 8, 2004, pp.11491160. 